ISSN electrónico: $1885-5210$

DOI: https://doi.org/10.14201/rmc20201615556

\title{
EL CINE COMERCIAL COMO RECURSO PARA LA DOCENCIA DE LA FARMACOLOGÍA
}

\section{Commercial cinema as a resource for Pharmacology teaching}

\author{
Magi FARRÉ ${ }^{1,2}$; Esther PAPASEIT ${ }^{1,2}$; Clara PÉREZ-MAÑÁ ${ }^{1,2}$; Josep-Eladi BAÑOS ${ }^{3}$ \\ ${ }^{1}$ Facultad de Medicina, Universitat Autònoma de Barcelona, Cerdanyola del Vallés. ²Servicio de Farmacología Clínica, Hospital \\ Universitari Germans Trias i Pujol-IGTP, Badalona. ${ }^{3}$ Facultad de Medicina, Universitat de Vic-Universitat Central de Catalunya. \\ Vic. (España).
}

e-mail: mfarre.germanstrias@gencat.cat

Las películas comerciales se utilizan cada vez con mayor frecuencia como método de enseñanza en muchas disciplinas universitarias, aunque su uso en las facultades de medicina es relativamente reciente. El cine se enmarca en el creciente interés por las humanidades médicas en muchos planes de estudio.

En medicina representan un recurso de interés, ya que se pueden ilustrar aspectos complejos de la medicina gracias al lenguaje cinematográfico, para facilitar la habilidad de los alumnos para observar y escuchar. Algunos autores consideran que, en algunos casos, la visión y discusión de una película puede ser equivalente al aprendizaje en las consultas reales con los pacientes. Temas como la relación médico-paciente, los dilemas éticos o la investigación son alguno de los temas más presentes en las películas comerciales.

Se emplea el término cinema-educación en referencia al uso de películas en formatos electrónicos (DVD entre otros) o recursos en línea, con películas de forma completa o parcial (clips), para educar a los estudiantes de medicina y los médicos residentes en algunos aspectos de la medicina. Recientemente, se han utilizado también las series médicas de televisión para el mismo objetivo.

Como parte de su relevancia docente cabe mencionar la publicación de su uso en artículos originales, metaanálisis y algunos libros. Hay que destacar la existencia de varias páginas web dedicadas al cine y medicina, algunos cursos universitarios y la existencia, desde hace quince años, de una revista dedicada casi monográficamente al tema (Revista de Medicina y Cine - The Journal of Medicine and Movies) ${ }^{1}$.

Las películas o partes de estas (en forma de clips cortos o secuencias) se usan generalmente para explicar algunos temas o seminarios, o se incluyen clips cortos de una película en algunas clases magistrales Es más excepcional que las películas sean la parte central de una materia o que toda ella se desarrolle únicamente visionando películas y trabajando sobre ellas.

Dentro de las distintas posibilidades, las películas comerciales pueden ser muy útiles como herramientas de enseñanza. Para garantizar unos resultados adecuados se requieren algunas consideraciones previas, tal como describen Baños y Bosch ${ }^{2}$ en un reciente artículo que recomendamos como lectura inicial. 


\section{EL CINE COMERCIAL COMO RECURSO PARA LA DOCENCIA DE LA FARMACOLOGÍA MAGI FARRÉ; ESTHER PAPASEIT; CLARA PÉREZ-MAÑÁ; JOSEP-ELADI BAÑOS}

Cine comercial en docencia de la Farmacología y la Farmacología Clínica

La Farmacología no es una materia fácil para los alumnos, es muy amplia y variada, con una parte memorística considerable para recordar los nombres de los medicamentos. No es de extrañar que el uso del cine en su docencia sea relativamente reciente. En la mayoría de los casos, las películas se han utilizado para profundizar en aspectos sociales del uso de fármacos o drogas y en el proceso de investigación clínica y/o sus aspectos éticos.

Nuestra experiencia se basa en el uso de diferentes películas comerciales en la enseñanza de la Farmacología / Farmacología Clínica en estudiantes de medicina. Una descripción más detallada de la experiencia se puede encontrar en tres de las referencias bibliográficas adjuntas $^{3,4,5}$

Algunas películas que hemos utilizado y pensamos especialmente útiles son: Awakenings (Despertares, 1990), Lorenzo's oil (El aceite de la vida, 1992), The fugitive (El fugitivo, 1993), Extreme measures (Al cruzar el límite, 1996), Miss Ever's boys (El experimento Tuskegee, 1997), The constant gardener (El jardinero fiel, 2005), Extraordinary measures (Medidas extraordinarias, 2010), Side effects (Efectos secundarios, 2013), Dallas Buyers Club (2013) y La fille de Brest (La doctora de Brest, 2016). Más recientemente hemos explorado el uso de episodios de series de televisión (House, MD o The Good Doctor) como alternativa al cine comercial estándar.

En general se han utilizado en el contexto de seminarios. Al inicio de estos, se entrega a los alumnos una descripción de los objetivos, se proyecta la película (en ocasiones para fomentar el seguimiento deben completarse algunas preguntas durante la proyección), una vez acabada se centran los temas de debate, se discuten los aspectos de interés y al final se resumen los temas más relevantes o de interés. En ocasiones unos días después se debe entregar un resumen escrito sobre la actividad y lo que se ha aprendido.
Como todos los métodos docentes, su uso puede ser complejo y se requiere tiempo para preparar y realizar la actividad. Encontrar la película adecuada no es fácil, pero en general los resultados de aprendizaje son muy satisfactorios.

Las películas comerciales pueden ser una herramienta útil para comprender algunos de los principios de la Farmacología y Farmacología clínica.

\section{Referencias}

1. García Moro M, García Sánchez JE, García Sánchez E, García Merino E. 15 años de la Revista de Medicina y Cine. Rev Med Cine [Internet]. 2019;15(1):1-2.

2. Baños JE, Bosch F. Using feature films as a teaching tool in medical schools. Educ Med. 2015;16(4):206-11.

3. Farré $M$, Roset PN, Bosch F, Baños JE. Puting clinical pharmacology in context: the use of popular movies. J Clin Pharmacol. 2004;44(1):30-6.

4. Farré $M$, Papaseit $E$, Pérez-Mañá $C$, Torrens $M$, Baños JE. Using popular movies in teaching: the case of pharmacology. In: Orefice C, Baños JE, editors. The role of humanities in the teaching of medical students. Barcelona: Fundació Dr. Antonio Esteve; 2018. p. 96-103. Monographs of the Esteve Foundation № 38 .

5. Baños JE, Bosch F, Orefice C, Farré M. Del interés de las películas comerciales en la docencia de la Farmacología: veinticinco años de historia. Actualidad en Farmacología y Terapéutica (AFT). 2017;15(4):313-7.

Resumen de la ponencia presentada en la Reunión científica La Revista de Medicina y Cine en los estudios de Ciencias de la Salud el día 7 de noviembre de 2019, en el marco del XXIV Congreso de la Sociedad Española de Educación Médica y V Congreso Hispano-Luso, celebrado en Salamanca del 6 al 8 de noviembre de 2019. 